\title{
Dissolution Universal Strategy Tool (DUST): A Tool to Guide Dissolution Method Development Strategy
}

Talia Flanagan ${ }^{1,2}$ and James Mann ${ }^{1 *}$

1Pharmaceutical Technology and Development, AstraZeneca, Macclesfield, Cheshire, UK.

${ }^{2}$ Present: UCB Pharma SA, Product Development, Chemin du Foriest, Belgium.

e-mail: james.mann@astrazeneca.com

\section{ABSTRACT}

Development of a dissolution method with suitable acceptance criteria is a key part of any oral drug products control strategy. As a key quality control test linked to safety and efficacy, dissolution strategy is often subject to extensive discussion during product development and with regulatory authorities during filing of the marketing application. A project review was recently performed at AstraZeneca to capture learning from dissolution method development and regulatory interactions from the last 10 years. The output of this review was distilled down to a few key messages that were critical to be considered when conducting dissolution method development. These key messages were then used as the building blocks for the Dissolution Universal Strategy Tool (DUST), that became part of the internal process at AstraZeneca for developing dissolution methods and acceptance criteria. The DUST can be used at all stages of product development and can be applied regardless of BCS Classification. The tool provides a common framework for cross-functional discussions, ensuring that project teams have a clear expectation of the role(s) of dissolution testing in drug product development, and that the understanding built during development will evolve into a suitable and clinically relevant QC test and acceptance criteria at time of submission which ensure the product will meet the patient's requirements.

KEYWORDS: Dissolution method development, dissolution acceptance criteria, clinically relevant dissolution, regulatory interactions

\section{INTRODUCTION}

issolution is widely recognised as a key quality test, and is used for several purposes during product development, including:

- Developing understanding of product performance;

- Assessing the likely performance of dosage forms in the patient;

- Evaluating the potential in vivo impact of changes to formulation or process; and

- Ensuring that batches of drug product are suitable for release to the clinic or market.

The connection between dissolution testing and in vivo performance has long been acknowledged $(1,2)$. With the advent of quality by design $(\mathrm{QbD})$, the pivotal role of dissolution testing in assuring product performance in vivo has gained increasing focus. The International Conference on Harmonisation (ICH) Q8R2 (3) describes the quality target product profile (QTPP) as: "A prospective summary of the quality characteristics of a drug product that ideally will be achieved to ensure the desired quality, taking into account safety and efficacy of the drug product," thus explicitly connecting clinical performance with drug product quality attributes and tests. Establishing the clinical relevance of dissolution tests and acceptance criteria in the QbD paradigm has been the subject of numerous publications, presentations, and discussions between industry and regulators (4-7). As a result, the expectations of a modern quality control (QC) dissolution test and the data needed to underpin its suitability have evolved significantly.

The dissolution method is a key component of any oral drug products QC strategy. The development of a robust and clinically relevant dissolution method requires a multitude of skill areas across a chemistry, manufacturing and control $(\mathrm{CMC})$ development group to work together. This is best facilitated by beginning with a deliberate

* Corresponding author. 
strategy, which is consciously reviewed and revised during the development process, to ensure that suitable data are generated in a timely manner, and that the resulting test and acceptance criteria meet the needs of all stakeholders, including regulatory authorities and the patient. To facilitate these cross-skill-area discussions and provide project teams a clear framework for thinking about the nature and role of dissolution testing in product development, a question-based tool for dissolution method development has been devised. At AstraZeneca, an internal portfolio review was performed to pull out key messages from recent regulatory interactions on dissolution methodology and acceptance criteria, and this was combined with external regulatory intelligence to produce the Dissolution Universal Strategy Tool (DUST). This article describes the key learnings distilled from the portfolio review, and the development and application of the DUST.

\section{THE PROCESS}

To leverage the regulatory experience of the organization, all late-stage development projects that had undergone some degree of regulatory interaction related to dissolution methodology and acceptance criteria were assessed. This totaled more than 10 projects with some having multiple regulatory interactions (e.g., end of phase 2, pre-new drug application [NDA], and during NDA review) and/or interactions with multiple agencies (e.g., United States Food and Drug Administration [FDA] and European Medicines Agency [EMA]).

To understand the scientific and strategic context for these interactions, information discussed with the project teams included physicochemical properties, formulation details, clinical study information, evolution of the dissolution method and acceptance criteria, main process and formulation risks, control strategy, and the regulatory strategy and feedback. For projects in the post-approval phase, the performance of the dissolution method in a commercial operation environment was also captured as well as any post-approval changes to the registered method or acceptance criteria. In addition, the teams were encouraged to reflect and to identify areas in the strategy where improvements or changes would have simplified the evolution of the dissolution strategy.

\section{REGULATORY FEEDBACK}

Upon reviewing recent regulatory interactions on dissolution, common elements in the regulatory feedback were identified across several projects. This provided a clear list of the agencies' expectations regarding information required to justify the dissolution method at time of submission. The information was requested to be submitted in the form of a method development report, as listed below:

- Solubility data across the physiological range and in any dissolution media used during the development (e.g., surfactant-containing media, biorelevant media). A calculation of the dose that could theoretically be solubilized and if sink conditions can be achieved should also be presented.

- A detailed description of the proposed method and justification for the selection of all developmental parameters including but not limited to: apparatus, dissolution medium, $\mathrm{pH}$, volume, agitation/ rotation speed, $\mathrm{pH}$, filter and sinker selection, and assay method. Data to support the type and amount of surfactant used (if applicable) should be provided. The feedback also stated that the dissolution profile. where possible. should be complete and cover at least $85 \%$ of drug release of the label amount or whenever a plateau (defined as no increase over three consecutive timepoints) is reached. The use of at least 12 samples per testing variable was also recommended; we have found that $n=12$ is necessary for key data or batches pivotal to the dissolution development story, but for early screening of methodologies a smaller sample, such as $n=6$, has been accepted.

- The complete dissolution profile including individual results, mean, and standard deviation should be provided; i.e., data at the proposed specification timepoint alone are not adequate for assessment of the method. The data should be reported as cumulative drug release with time; in the authors experience it is also a good idea to present an infinity spin (e.g., increase rotation speed to 250 rpm after the final timepoint) to demonstrate that all drug that could dissolve has dissolved.

- All testing that has been conducted to demonstrate the discriminating capability of the selected dissolution test should be presented. In general, this should compare the reference or target product versus test products that are intentionally manufactured with meaningful variations for the most relevant clinical manufacturing variables. The feed back suggested $\pm 10-20 \%$ change relative to the specification setpoint or range of these variables. In our experience, wider variations 
can often be presented, particularly for process parameters or changing of grades of excipients as part of the $\mathrm{QbD}$ product design or process stretch work; however, variants where excipients are deleted or replaced and dramatic changes to the process train are less likely to be acceptable. Though not specifically mentioned in the feedback, a link to the clinical studies used to demonstrate an in vitro in vivo relationship (IVIVR) (e.g., definition of a dissolution safe space (8)) is important to demonstrate appropriate discriminating capability of the method and set clinically relevant acceptance criteria.

- Data should be presented to support the robustness of the proposed method and the validation of the detection method. Robustness can focus on factors such as but not limited to temperature, paddle speed, media volume, sinker type, and $\mathrm{pH}$.

- When setting acceptance criteria for the proposed drug product, release profile data (i.e., as a minimum for an immediate release product: at 15, 20, 30, 45 , and 60 minutes) from the clinical batches and primary (registration) stability batches should be used for the setting of the dissolution acceptance criteria of both sampling timepoint and limit. They also state that the selection of the timepoint should be when $Q=80 \%$ dissolution occurs, as the preference for a QC method does appear to be a method where full release is possible. It is also stated that, for a slow dissolving immediaterelease (IR) product or a product that includes a Biopharmaceutics Classification System (BCS) 2 or 4 compound, a 2-point acceptance criteria may be needed for the product, with the first timepoint during the initial phase of the dissolution (i.e., 15$20 \mathrm{~min}$ ) and the second timepoint where $Q=80 \%$ occurs. They also state that the acceptance criteria should be based on data with $n=12$ and that final determination of acceptability of acceptance criteria will occur during the final submission review.

The regulatory information requirements were observed to be consistent across projects and mostly across agencies, and so were incorporated in development of the DUST to ensure that no gaps existed in the expected data package by the time of submission.

\section{KEY MESSAGES BASED ON PROJECT EXAMPLES}

Following the review exercise, six key messages were identified that are general good practice when developing a dissolution strategy. These key messages are described and exemplified below.

Do not fixate too early on one method - use a suite of tests to develop mechanistic understanding of formulation, process, and in vivo risks.

The dissolution test has many different roles to play during development, so it is unreasonable to expect that one set of method conditions will fit all the project needs. However, project teams can sometimes be reluctant to move away from the initial QC method used for early batch release, which is included in the Investigational New Drug (IND) application, even though the formulation and manufacturing process will continue to evolve or even change entirely during development. Using a suite of dissolution tests (e.g., different media, apparatus, conditions, etc.) can be perceived as indecision on the part of the project team or challenged as a waste of resources. However, to build understanding of the in vivo performance and to fully characterize the in vitro performance of the product and manufacturing process, it is essential to assess dissolution under a range of conditions. This is particularly important when deliberate changes are being made to challenge product and process robustness (for example during Design of Experiment [DoE] studies), but also to ensure that batch-to-batch performance is well understood.

Pepin et al. (9) describe the use of physiological-based pharmacokinetic (PBPK) dissolution modelling to justify the dissolution release acceptance criteria for lesinurad. Lesinurad is a BCS class 2 weak acid used in treatment of gout. During development, an over-granulated batch was manufactured (batch "ELAB"), which showed reduced $C_{\max }$ and $A U C$ compared to a standard clinical batch. Geometric mean ratios for $\mathrm{C}_{\max }$ and $A U \mathrm{C}_{\infty}$ versus the reference lot (batch "12A015") were 80.0\% (90\% confidence interval [Cl]: 68.2-93.8\%) and $88.1 \%(90 \%$ $\mathrm{Cl}$ : 79.9-97.2\%), respectively, and $t_{\max }$ was delayed by approximately 1 hour. The registered QC dissolution test for lesinurad ( $\mathrm{pH} 4.5$ acetate buffer plus $1 \%$ sodium lauryl sulfate [SLS], $900 \mathrm{~mL}$, USP apparatus 2, $75 \mathrm{rpm}$ ) reflects the in vivo results, as batch ELAB shows slow and incomplete release under these conditions (Fig. 1). However, earlier in 
development a simple aqueous buffer method was used (pH 6.8 phosphate buffer, no surfactant, USP apparatus 2 $75 \mathrm{rpm})$. Despite the absence of surfactant, this method did not substantially discriminate for batch ELAB, showing a similar release profile to a standard clinical batch (Fig. 1). This exemplifies the importance of evaluating dissolution performance under multiple conditions while process understanding is being developed, as the early release method would have failed to detect the altered in vivo performance of the over-granulated batch.
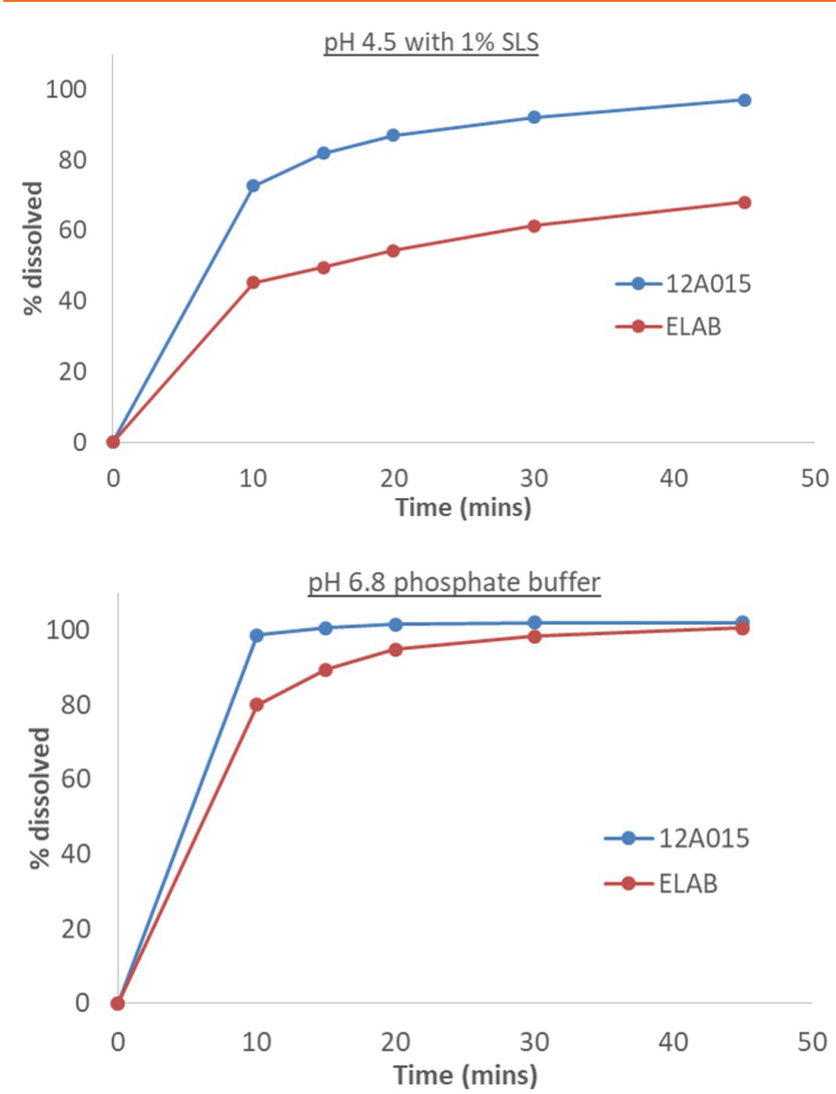

Figure 1. Lesinurad dissolution profiles in $\mathrm{pH} 4.5$ plus $1 \%$ SLS and at $\mathrm{pH}$ 6.8, for batch "ELAB" (over-granulated batch) plus a standard clinical batch (12A015). SLS, sodium lauryl sulfate.

\section{Use a mechanistic and risk-based approach to dissolution}

When developing a dissolution testing strategy, it is essential to consider which mechanisms are controlling dissolution of the drug from the formulation, and which formulation and process parameters could affect these (i.e., present a risk to dissolution performance). Without this mechanistic understanding as the basis for method development, there is the risk that the dissolution method could give false reassurance of similar in vitro performance, even if significant process changes have been made. These are several examples in the literature that illustrate the importance of risk-based thinking as the foundation in dissolution method development. Dickinson et al. (4) describe a five-step process to establish a clinically relevant dissolution test to ensure suitable clinical quality of a drug product (i.e., that the drug product delivers the required safety and efficacy). This process begins with a quality risk assessment (QRA) to determine the drug substance, drug product, and manufacturing process attributes that present the highest risk for in vivo dissolution. In a recent Innovation and Quality (IQ) Consortium white paper (6), mechanistic understanding of drug product dissolution, and identification of formulation and manufacturing process parameters that can impact dissolution, are identified as critical first steps in developing a clinically relevant dissolution method, which is defined as a dissolution method with an established link to in vivo performance. This element is also linked to the key message below, as the next step is to characterize the impact of the risks identified on in vivo drug product performance.

\section{Characterize the in vivo performance impact of your highest process and formulation risks}

Assessing the impact of changes to the extent and rate of dissolution (e.g., caused by formulation and process changes) on in vivo product performance is a key element of dissolution method development and establishment of the QC strategy. This topic has been the subject of numerous publications and conference presentations from industry and regulatory authorities $(4,6-8,10-12)$. Establishing the link between in vitro dissolution and clinical product performance should be a key consideration for project teams, as this underpins selection of a relevant test with an appropriate degree of discriminatory power versus in vivo performance, and this is an important link to discuss with regulatory authorities when justifying the method and acceptance criteria. Hermans et al. (6) and Dickinson et al. (4) describe detailed approaches to establish the link between in vivo and in vitro performance as part of the development of clinically relevant dissolution tests and acceptance criteria. Often, this will involve the generation of clinical relative bioavailability data on relevant drug product process/formulation variants; several examples have been published and presented for specific drug products $(4,6,8,10,13,14)$. Recently, in silico PBPK absorption modelling has emerged as a key tool in development of clinically relevant specifications to mechanistically explore and describe the link between the in vitro and in vivo data $(9,15)$. 


\section{Consider the nature and level of discrimination required in context of the wider QC strategy}

The QC dissolution test used for routine batch release is only one element of the wider control strategy and needs to be considered in this context when selecting a final $Q C$ method. It is highly unlikely that a single $Q C$ dissolution method will be able to detect all potential failure modes; however, this is not necessary, as some of these will be eliminated or constrained through controls earlier in the manufacturing process (e.g., by input material controls, in process controls [IPCs], etc).

Fostamatinib is an orally dosed phosphate ester prodrug of the active moiety R406. Phase 2 studies in rheumatoid arthritis were performed using an early microcrystalline cellulose (MCC)-based formulation. Low and variable dissolution in $0.1 \mathrm{~N}$ hydrochloric acid $(\mathrm{HCl})$ media was observed for some batches of the 100-mg strength of this formulation and was attributed to gelling of the drug substance under acidic conditions, which was exacerbated by high drug loading (13). This failure mode was shown to have some in vivo relevance in a clinical relative bioavailability study, as batches with very low dissolution in $0.1 \mathrm{~N} \mathrm{HCl}(\sim<50 \%$ release) exhibited altered pharmacokinetics (13). However, no effect was detected in $\mathrm{pH} 7.4$ media, which had been used as an early QC method. A new formulation was developed to mechanistically overcome the gelling phenomenon, using sodium bicarbonate to aid tablet disintegration under acidic conditions and changing to a soluble filler (mannitol-based). For this formulation, dissolution under acidic conditions was less sensitive than dissolution at pH 7.4 for relevant failure mechanisms (Fig. 2) (13). The project team selected a dissolution method at $\mathrm{pH}$ 7.4 for the mannitol-based formulation, as gelling (the only failure mechanism with known in vivo impact) was robustly controlled by other elements of the control strategy (i.e., the formulation composition).

Other examples of this could include polymorphic form, if this is controlled elsewhere in the manufacturing process or has been demonstrated not to be altered within the operating ranges allowed by the control strategy, or drug substance particle size if this is controlled at the input material stage.

\section{Method and acceptance criteria go hand-in-hand - discriminatory power has an impact on specification risk}

When selecting a final QC dissolution method, the discriminatory power of the dissolution test needs to be considered in the context of setting appropriate release acceptance criteria. There is a regulatory expectation that the dissolution acceptance criteria will be set to ensure complete release, i.e., a $Q$ value of $80 \%$. Dissolution is different from other release tests such as assay, as the test does not measure an absolute property of the formulation (e.g., the amount of drug substance in a tablet). Rather, the rate and extent of release observed in a dissolution test is a function of the media and testing conditions as well as the formulation properties. This interplay between method conditions, the discriminatory power of a dissolution test and the ability to pass acceptance criteria that would not unnecessarily fail batches that would deliver the required in vivo performance needs to be given active consideration when selecting the dissolution test for $Q C$ release.

Selecting a method with appropriate discriminatory power is a balancing act that requires consideration of several factors. A clinically relevant dissolution test should be able to discriminate for drug product failure mechanisms that can impact performance in the patient to ensure that all batches released will have equivalent in vivo performance. However, in a commercial setting, the method will be exposed to more sources of variability than during development (e.g., manufacturing process, input materials, test factors) - an over-discriminatory test plus tight acceptance criteria could lead to supply problems, potentially leading to failure of clinically acceptable batches.

Flanagan (10) describes an example of this for a BCS 4 compound, formulated as an immediate release tablet. An IVIVR study was undertaken during development, where tablet variants incorporating the highest risk formulation and process variants were dosed to healthy volunteers. All batches gave bioequivalent exposure to standard tablets, and a dissolution safe-space was established. The selected dissolution method was overdiscriminating with respect to in vivo performance (Fig. 3). A dissolution acceptance criterion of $Q=70 \%$ at 45 minutes was initially proposed, which is well-within the established region of bioequivalence based on the clinical IVIVR study. This was accepted in all territories except the US, where an additional acceptance criterion of $Q=80 \%$ at 60 minutes was requested, based on a requirement to show complete release (i.e., $>80 \%$ ). During commercial manufacture, additional sources of variability, primarily in drug substance, were encountered. Statistical modelling highlighted that if dissolution performance shifted by $5 \%$ (e.g., due to method transfer to another site), the US acceptance criteria would end up failing a significant number of clinically acceptable batches (approximately 1 


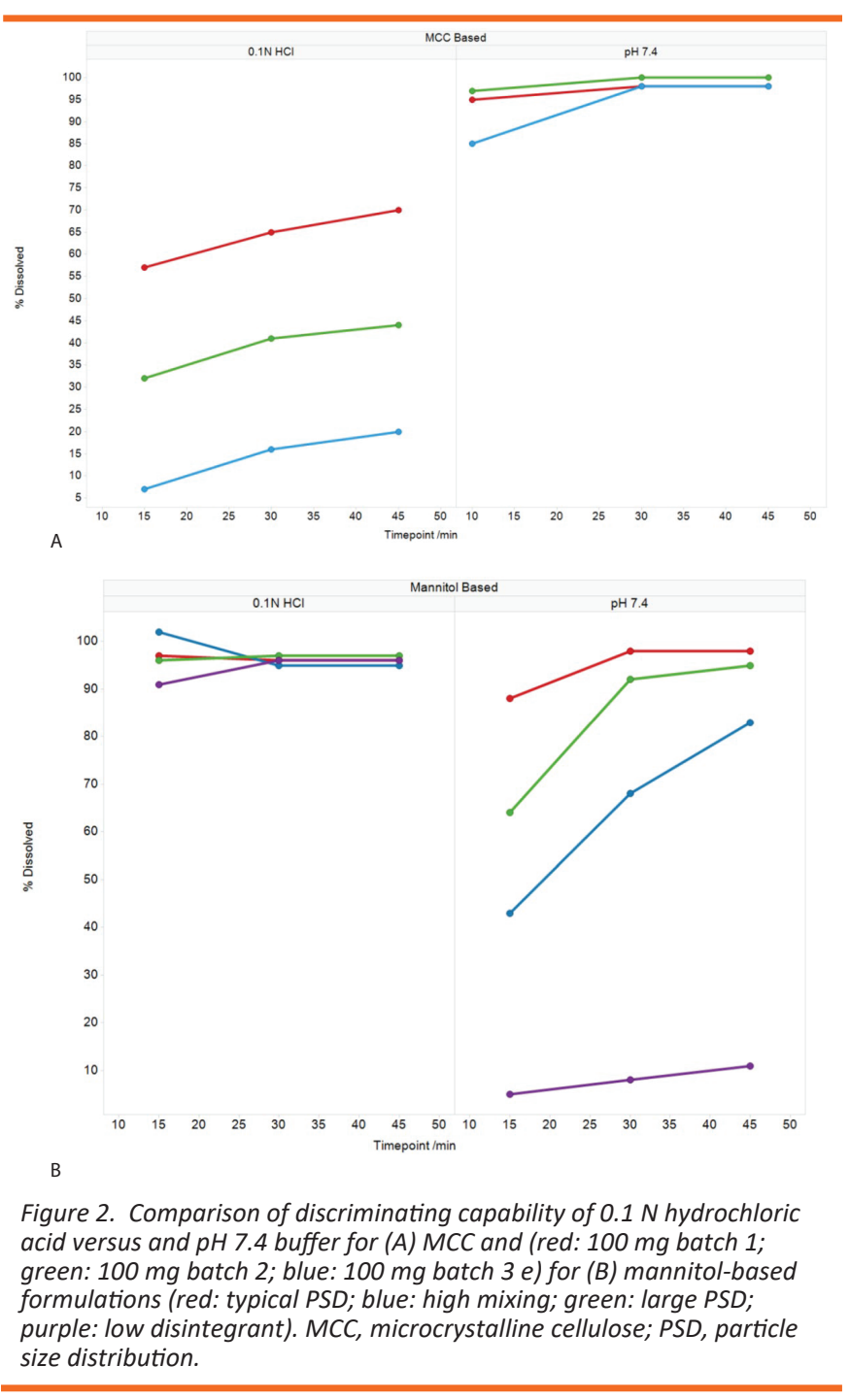

in 14) (Fig. 3). Based on this, the US acceptance criteria was successfully renegotiated to $Q=75 \%$ at 60 minutes - this significantly reduced the risk of failing clinically acceptable batches for this product $k 1$ in 300 batch failures).

\section{Develop and manage your dissolution knowledge base well throughout the project}

Several projects received requests for large volumes of historical dissolution data during regulatory review. Typically, the information requests would require provision of the complete raw dissolution data, lot number, manufacturing parameters, dissolution conditions used, individual and average values of percent dissolved at each time point, and $f_{2}$ values where applicable. The data request included data from formulation development, formulations used at all stages of clinical development,
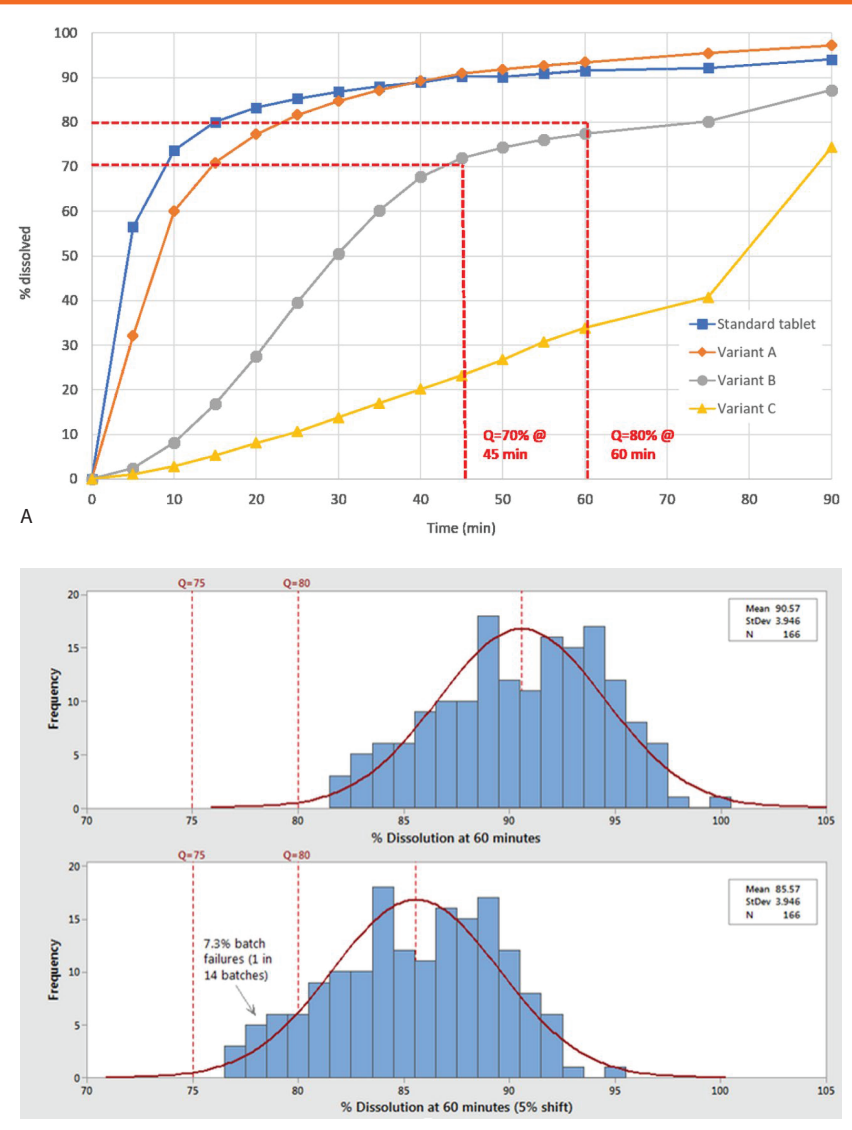

B

Figure 3. Dissolution profiles for example compound in the highly discriminatory $Q C$ release method and predicted batch failure rates for $Q=80 \%$ at 60 minutes acceptance criteria. Panel A shows the dissolution profiles for the tablet variants and the standard tablet dosed in the IVIVR study, with region of bioequivalence being seen from varient $C$ to standard tablet. Panel $B$ shows the distribution of dissolution in the same dissolution method for percent dissolved at 60 minutes (top), and a simulation of predicted batch failure rates with a $5 \%$ shift to illustrate the risk of failing clinically acceptable batches (bottom). IVIVR, in vitro in vivo relationship; StDev, standard deviation. the proposed commercial formulation, any variants tested either in vitro or in vivo, formulations from process DoEs and any pilot or commercial-scale batches produced to date.

These broad requests could be challenging to fulfil in a timely manner, due to how the project team had been collating dissolution data at different stages of development (e.g., across many separate electronic lab notebook records). This resulted in project teams spending large amounts of time collating, transcription checking, and formatting data to send to the agencies, under significant time pressure during the review period. These difficulties also highlighted that the project teams were not taking the opportunity to look at their dissolution data holistically across the history of the project, which could lead to learning opportunities being missed. 
In response to this, it was recommended that project teams take early ownership of the dissolution data and associated meta-data for the batches tested and construct central databases from project inception that allow experimental references and transcription checking or seamless data transfer to be built in. Visualisation tools such as Spotfire Analytics (TIBCO Software, Inc.) have shown to be very powerful tools in aiding management of dissolution data. This has made transfer of dissolution data in response to these requests easier to fulfil and has facilitated discussion of the complete dissolution history within projects, enabling any new data generated to be put into appropriate context.

\section{DEVELOPMENT OF DISSOLUTION UNIVERSAL STRATEGY TOOL (DUST)}

To consolidate the learning described above in a format that could be used by project teams seeking to develop their dissolution strategy, a question-based dissolution strategy tool was developed, i.e., DUST. The aims of the tool were to:

- Facilitate structured discussion on dissolution strategy and generate common expectations on the role of dissolution testing in the project team, including the link to the patient; and

- Drive a consistent approach across projects to dissolution method development within the organization, ensuring application of the latest regulatory learning and current expectations of the dissolution test.

In contrast to other specification tests, the dissolution test is a multi-disciplinary test impacting many areas of the $\mathrm{CMC}$ development team, by nature of the test being related to multiple aspects of product performance and ultimately safety and efficacy. Reflecting this, the key participants in the DUST process would normally include representatives from analytical, biopharmaceutics, formulation, materials science, and solid state and drug substance process chemistry. The need for input from analytical and biopharmaceutics representatives is due to the close link between the analytical method, measured physicochemical properties, and the in vivo performance. This close tie is also mirrored in regulatory agency reviews of dissolution methods. It is also often the case in modern drug development that the biopharmaceutics representative is utilising the in vitro dissolution as a key input to in silico PBPK modelling and ensuring the method is providing the necessary clinical relevance. The formulation scientist needs to be involved in identifying key process and manufacturing risks that can impact dissolution performance and to produce batches of product with meaningful variations to test the discriminatory nature of the method. The material scientist needs to bring understanding of the excipient effects along with the particle properties of the drug substance. The solid-state scientist needs to bring the understanding of the polymorphic form or salt characteristics and the propensity for any change of these during dissolution. Finally, the drug substance scientist needs to ensure the link between particle size distribution of the active pharmaceutical ingredient (API) (and other physical property descriptors) and the dissolution method is understood, and that a meaningful variation in the range of properties is manufactured to allow the discriminatory nature of the method to be tested and ultimately feed into the input drug substance specification. The frequency of the DUST meeting is informal and can be driven based on key project milestones and/or significant changes in the formulation or API.

The DUST itself is a simple question-based tool, consisting of 10 questions and considerations relevant to each question. The project team uses the DUST questions to highlight identified knowledge gaps, assign actions to fill the gaps, and document decisions taken by the team with regard to dissolution strategy. The process was designed to be simple, flexible, and informal, so DUST may be used in an agile manner without becoming a cumbersome resource drain on project teams. Approximately 50 DUST sessions have been completed in the 3 years since the tool was launched. The tool has also proved invaluable in evaluating data packages and developing strategies quickly when drug molecules are in-licensed.

The DUST questions are summarised and described in more detail below. The questions have been designed to be relevant to different stages of development. When DUST is used early in development, the expectation is that the earlier questions (Q1-Q4), which focus more on product understanding, are the most important. The later questions (Q5-Q10), which focus more on operability of the method, become more important as you near transfer to a commercial site. Application of DUST should result in a:

- Clear understanding of dissolution-related risks to product performance, underpinning design and optimisation of the drug product and manufacturing process;

- Clear linkages between the selected QC dissolution 
method and acceptance criteria and in vivo product performance (i.e., clinical relevance);

- QC dissolution method(s) with appropriate discriminatory power for relevant failure mechanisms in the context of the overall control strategy; and

- Clear justification for the selected QC method and acceptance criteria to facilitate discussions with regulatory authorities, which should minimise the risk of late changes to dissolution method or of $\mathrm{CMC}$ being on the critical path for approval.

\section{Question 1}

Q1 is What in vivo performance is expected from my drug product based on biopharmaceutical and formulation properties? This question is designed to get project teams thinking about the design intent for the drug product, from the perspective of achieving the required efficacy and safety in the intended patient population. In answering this question, the team should understand the API physicochemical properties, the target patient population and therapy area, formulation properties including the route of administration and intended in vivo release profile, expected potencies and dosing regime, and if any medicines are likely to be coadministered. Some of the information can be leveraged from the QTPP, additionally in silico modelling can be utilised. Answering of the question can act as reminder and as a prompt for the later questions, by ensuring continued focus on the patient (rather than purely on the more traditional analytical considerations). Potential impacts on dissolution strategy that can arise from Q1; for example, the relevance of low $\mathrm{pH}$ to the patient population; the selection of the dissolution apparatus can be influenced by the choice of formulation and its mechanism of release; also, if the product will be dosed fed or in the presence of proton pump inhibitors then additional dissolution studies may be needed.

\section{Question 2}

Q2 is How does my expected in vivo performance link to my expected dissolution performance based on biopharmaceutical and formulation properties? This question is designed to take the input from Q1 and begin to translate that into in vitro experiments and early dissolution targets for formulation design. Considerations for Q2 are the pKa of the API; the solubility of the API across the physiological $\mathrm{pH}$ range and in biorelevant media; the definition of sink conditions in potential release media, the formulation type, and the role of any functional excipients; any preclinical/clinical data or in silico modelling data that can be leveraged; and finally, information on the API form and particle size strategy. Potential impacts on the dissolution strategy could be identification of a precipitation risk that needs to be investigated via a pH-shift methodology or the TNO Intestinal Model (TIM-1), a requirement for a surfactant, or potential for supersaturation (e.g., amorphous solid dispersion), for which the type of supersaturation and duration needs to be explored.

\section{Question 3}

Q3 is What are the key risks based on the drug product and manufacturing process that could impact dissolution? This question is designed to make the team consider all potential parameters from the formulation, the process and the API that can affect dissolution (i.e., shifting the focus from patient-related risks in Q1 and Q2 towards product and process risk factors). The answers here are normally leveraged from the QRA for the product. Examples of impacts on strategy can be to evaluate API particle size variants, assess impact of form change or salt switches, evaluate processing parameters related to blending, granulation, lubrication, compression, coating, and changes in functional excipient grades and levels such as disintegrants. It is also suggested to leverage in silico modelling during this stage.

\section{Question 4}

Q4 is Based on the previous answers - what dissolution methodologies will be evaluated, and which methods do you need to employ at the current stage of development? Also, state the purpose of the method. Areas to focus on during the answering of this question is choice of apparatus, medium including $\mathrm{pH}$, concentration, volume, surfactant type/level and ionic/osmotic strength, sampling timepoints, hydrodynamic conditions, and use of biorelevant media and tests including more advanced tools, such as TIM-1. A careful consideration of what batches should be tested in what methods should be made. Potential outputs here include agreeing to use a high sink method for release during clinical development but also carry more discriminatory methods throughout development that would allow a later switch as product and clinical understanding grows. It may also highlight that a pH shift method, non-compendial apparatus or an advanced dissolution tool should be part of the cascade for key development batches. 


\section{Question 5}

Q5 is Based on your current product and process understanding and evolving control strategy, what do you need the dissolution test(s) to discriminate for? The idea here is that, if Q3 highlights all the potential failure mechanisms that you might need the dissolution method to control for, then Q5 focuses the discussion on the use of the dissolution test as part of the evolving control strategy. This question is expected to come into focus at a later stage in development, when greater understanding of critical quality attributes has been established and an early control strategy is in place. Considerations could include feedback from any bioequivalence or relative bioavailability data, i.e., does the dissolution method allow for the outcome of those studies to be accurately predicted (e.g., solution versus suspension during early development), or has the particle size strategy or API form strategy changed such that they are controlled earlier in the process than at finished product testing (e.g., either via milling/micronization or crystallization). In addition, excipient levels and acceptable process ranges may now be established, and the dissolution method is no longer needed to discriminate across as wide a range of process parameters for batch release. It is normally during the review of Q5 that it becomes apparent if an IVIVR study is required, where testing variants with differing release rates in the clinic are used to facilitate selection of a clinically relevant dissolution method and acceptance criteria. Additionally, the use of advanced dissolution tools, preclinical studies, and in silico PBPK modelling can aid in the dissolution method selection process.

\section{Question 6}

Q6 is At your current stage of development, what method/technique and acceptance criteria will be used to release clinical batches? The aim of this question is to agree how the quality of the batch will be assured to give the required performance of the product for the patient. The key considerations for Q6 are the stage of development and the associated regulatory expectations for the stage of development and the risk level associated with product.

\section{Question 7}

Q7 is an extension of Q6: Based on the previous question, what method(s) and acceptance criteria will be used to monitor drug performance on stability? This is often the same answer to Q6, but on occasion a second method may be being used to collect key stability timepoints to facilitate a switch later in development or if only certain methods can access dissolution instability.

\section{Question 8}

Q8 is Can any of the existing dissolution tests be used as a QC method (i.e., document acceptable discrimination, extent of release, and reproducibility to provide an appropriate regulatory acceptance criteria)? Points to consider are the statistics of the method such as batchto-batch variability and within-batch variability, process capability in relationship to the proposed acceptance criteria with an acceptable number of batches studied, a link to an IVIVR if available, sink conditions, and apparatus requirements. The key here is to ensure that a coherent method development story exists, enough batches have been tested to give confidence in the method selection, and that the proposed method and acceptance criteria can exclude failed bioequivalence batches or accessible variants with slow dissolution rates.

\section{Question 9}

Q9 is the key question about method operability: Does the proposed QC and stability method exhibit acceptable robustness and ruggedness to enable effective method transfer and long-term operability in a QC environment? This question is usually addressed as the project nears the selection of the final method and needs to consider filter supply, media preparation, component suppliers, sampling methods, automation, deaeration techniques, intermediate precision, and the continuous process verification strategy. This leads to a robustness assessment, where the impact of different sinkers, surfactant grades, method of degassing, and alternative filter suppliers, etc., is conducted to ensure that the method is suitable to undergo multiple method transfers without issues.

\section{Question 10}

Q10 is Are there any aspects of your dissolution strategy resulting from completing this exercise that would benefit from consultation with internal advisory or external regulatory bodies? This is designed to allow project teams that have struggled to reach a consensus or do not have enough technical knowledge to answer the questions to access expert advice either from internal technical or regulatory experts, statistical analysis support, clinical input, or through some regulatory interaction with the agencies. Engagement with the regulatory agencies on the dissolution strategy well in advance of a filing date is actively encouraged within AstraZeneca as the risk of a late change in dissolution method can result in repeated work just prior to filing or during review, which can end up with a switch to a method where there is a lack of experience and robustness information. 


\section{CONCLUSIONS}

We have presented DUST, a question-based tool which acts as a framework for dissolution strategy development based on insight from the experiences of negotiating meaningful dissolution acceptance criteria with regulatory agencies and the information needed to support those discussions.

The DUST tool can be used at all stages of product development and can be applied regardless of BCS classification to provide up-to-date guidance to project teams. The DUST tool has been successfully used on more than 50 occasions in the last 3 years on most AstraZeneca projects that involve dissolution methods. A modified version with the same questions, but adjusted considerations has been developed for paediatric projects (pDUST). In addition, DUST has been utilised for controlled-release parenterals when developing in vitro release methodology, and the framework has proven useful for these non-oral examples. The tool provides a common framework for cross-functional discussions, ensuring that project teams have a clear expectation of the role(s) of dissolution testing in drug product development, and that the understanding built during development will evolve into a suitable and clinically relevant QC test and acceptance criteria at time of filing.

\section{ACKNOWLEDGEMENTS}

The authors would like to acknowledge David Holt for his assistance in the design of DUST process.

\section{CONFLICT OF INTEREST}

The authors are employees of AstraZeneca and the work was conducted as part of their employment.

\section{REFERENCES}

1. Prasad, V. K.; Shah, V. P.; Knight, P.; Malinowski, H.; Cabana, B. E.; Meyer, M. C. Importance of media selection in establishment of in vitro-in vivo relationships for quinidine gluconate. Int. J. Pharm. 1982, 13, 1-7. DOI: 10.1016/0378-5173(82)90137-5.

2. Dissolution Testing of Immediate Release Solid Oral Dosage Forms; Guidance for Industry; U.S. Department of Health and Human Services, Food and Drug Administration, Center for Drug Evaluation and Research (CDER), U.S. Government Printing Office: Washington, DC, 1997.

3. International Conference on Harmonisation of Technical Requirements for Registratin of Pharmaceuticals for Human Use. Pharmaceutical Development, Q8(R2); Current Step 4 version; ICH Harmonised Tripartite Guideline: Geneva, Switzerland, August 2009.

4. Dickinson, P. A.; Flanagan, T.; Holt, D.; Stott, P. W. Clinically relevant dissolution for low-solubility immediate-release products. In Poorly Soluble Drugs; Webster, G. K.; Bell, R. G.; Jackson, J. D.; Eds.; Jenny Stanford Publishing, 2016; pp 535-76.

5. Suarez-Sharp, S. The future of clinically relevant dissolution testing in drug product development - a regulatory perspective. 5 Years of progress in Oral Biopharmaceutics - IMI OrBiTo project and global biopharmaceutics research. Presented at OrBiTo $6^{\text {th }}$ Annual F2F Meeting, Granta Park, Cambridge, UK, September 17-18, 2018. https://www.apsgb.co.uk/wp-content/ uploads/2019/04/OrBiTo-6th-Annual-F2F-Meeting-summaryreport.pdf (accessed June 25, 2019).

6. Hermans, A.; Abend, A. M.; Kesisoglou, F.; Flanagan, T.; Cohen, M. J.; Diaz, D. A.; Mao, Y.; Zhang, L.; Webster, G. K.; Lin, Y.; Hahn, D. A.; Coutant, C. A.; Grady, H. Approaches for establishing clinically relevant dissolution specifications for immediate release solid oral dosage forms. AAPS J. 2017, 19, 1537-1549. DOI: 10.1208/ s12248-017-0117-1.

7. Suarez-Sharp, S.; Cohen, M; Kesisoglou, F.; Abend, A.; Marroum, P.; Delvadia, P.; Kotzagiorgis, E.; Li, M.; Nordmark, A.; Bandi, N.; Sjögren, E.; Babiskin, A.; Heimbach, T.; Kijima, S.; Mandula, H.; Raines, K.; Seo, P.; Zhang, X. Applications of clinically relevant dissolution testing: workshop summary report. AAPS J. 2018, 20, 93. DOI: 10.1208/s12248-018-0252-3.

8. Dickinson, P. A.; Lee, W. W.; Stott, P. W.; Townsend, A. I.; Smart, J. P.; Ghahramani, P.; Hammett, T.; Billet, L.; Behn, S.; Gibb, R. C.; Abrahamsson, B. Clinical relevance of dissolution testing in quality by design. AAPS J. 2008, 10, 380-390. DOI: 10.1208/ s12248-008-9034-7.

9. Pepin, X. J.; Flanagan, T. R.; Holt, D. J.; Eidelman, A.; Treacy, D.; Rowlings, C. E. Justification of drug product dissolution rate and drug substance particle size specifications based on absorption PBPK modeling for lesinurad immediate release tablets. Mol Pharm. 2016, 13, 3256-3269. DOI: 10.1021/acs. molpharmaceut.6b00497.

10. Flanagan, T. Developing clinically relevant specifications: case studies and future directions. IFPAC Annual Meeting; Washnigton D. C., USA, February 27-March 2, 2017.

11. Pope-Miksinski, S. Quality by design and clinical relevance. IFPAC Annual Meeting; Washnigton D. C., USA, February 27-March 2 2017.

12. Kesisoglou, F. The role of modeling and simulation in quality by design and clinically relevant dissolution specifications. IFPAC Annual Meeting; Washnigton D. C., USA, February 27-March 2, 2017.

13. Flanagan, T.; Martin, P.; Gillen, M.; Mathews, D.; Lisbon, E.; Kruusmägi, M. Effects of ranitidine (antacid), food, and formulation on the pharmacokinetics of fostamatinib: results from five phase I clinical studies. Eur. J. Clin. Pharmacol. 2017, 73, 185-195. DOI: 10.1007/s00228-016-2156-4.

14. Kesisoglou, F.; Hermans, A.; Neu, C.; Yee, K. L.; Palcza, J.; Miller, J. Development of in vitro-in vivo correlation for amorphous 
solid dispersion immediate-release suvorexant tablets and application to clinically relevant dissolution specifications and in-process controls. J. Pharm. Sci. 2015, 104, 2913-2922. DOI: 10.1002/jps.24362.
15. Li, M. Building clinical relevance into QbD: The role of biopharmaceutics from a regulatory perspective. Presented at IFPAC Annual Meeting; Washnigton D. C., USA, February 27March 2, 2017. 